\title{
The Heavy Ion Beam Probing Development for WEGA Stellarator
}

\author{
L. I. Krupnik ${ }^{1}$, G.N. Deshko ${ }^{1}$, A.I. Zhezhera ${ }^{1}$, A.A. Chmyga ${ }^{1}$, A.D.Komarov ${ }^{1}$, A.S. Kozachek ${ }^{1}$ \\ A.V. Melnikov', S.V. Perfilov ${ }^{2}$, M. Otte ${ }^{3}$, M. Shubert ${ }^{3}$ \\ ${ }^{1}$ NNC "KhIPT”, Kharkov, Ukraine ${ }^{2}$ RRC "Kurchatov Institute”, Moscow, Russia, \\ ${ }^{3}$ IPP Max-Plank Institute, Greifswald, Germany
}

\begin{abstract}
.
The conceptual design for a Heavy Ion Beam diagnostic (HIBP) for the stellarator WEGA in Greifswald (Germany) is developed to provide the measurements of the radial profiles of the electric plasma potential, density and their fluctuations. Calculations of probing $\mathrm{Na}+$ beam trajectories were done for the various WEGA diagnostics ports with $\mathrm{B}_{0}=0.5 \mathrm{~T}$. They show that satisfactory access may be possible for $\mathrm{C}+-\mathrm{C}^{*}$ port combinations.

\section{Introduction}

In the last years the HIBP diagnostic becomes rather popular and was developed for same magnetically confined fusion devices. It is due to that HIBP has the possibility to perform measurements of several main plasma parameters in improved confinement regimes. Some of them are linked to electric and magnetic fields, the measurements of which are unique capability for this diagnostic. Essential potentialits inherent in HIBP are investigations of the plasma parameters and their fluctuayions from center to deep periphery of plasma column.[1]

Radial electric fields play a key role in plasma turbulence and confinement. The best performance of devices has been obtaind in plasma regimes where ExB-shear stabilizing mechanisms are likely to play a role. Stellarators are inherently flexible devices, very well suited for the investigation of the complex phenomenology, that interrelates electric field, instabilities,magnetic configurations and transport
\end{abstract}

\section{Basic Principles of the HIBP method.}

Heavy Ion Beam Probe (HIBP) is based on the ion beam injection across the confinement magnetic field. Fig.1. When the beam of high-energy single charged ions passes through the plasma, some of the beam ions ionize, predominantly by the electrons. The ionization takes place along the full path of the beam in the plasma. Because of their higher charge state, the secondary ions deviate from the primary beam and form a broad fan of ions leaving the plasma. The secondary ions that enter the detector aperture, originate from a small part of the primary beam in the plasma, called the sample volume, which has typical dimensions 
of $(0.5-1) \mathrm{cm}^{3}$. The energy difference between the secondary ions leaving the plasma and the primary ions is equal to the electric potential $\varphi$, at the sample volume. The intensity of the secondary beam is proportional to the electron density, $\boldsymbol{n e}$, in the sample volume. The toroidal velocity of the secondary beam in the detector reflects the poloidal component of magnetic vector potential (poloidal magnetic field/plasma current density). The position of the sample volume can be rapidly changed by redirection of the probing beam with electrostatic sweep plates or by change of the probing beam initial energy. HIBP is performed a continuously measurements in time, which provide a high temporal resolution limited only by the possibilities of the acquisition electronics

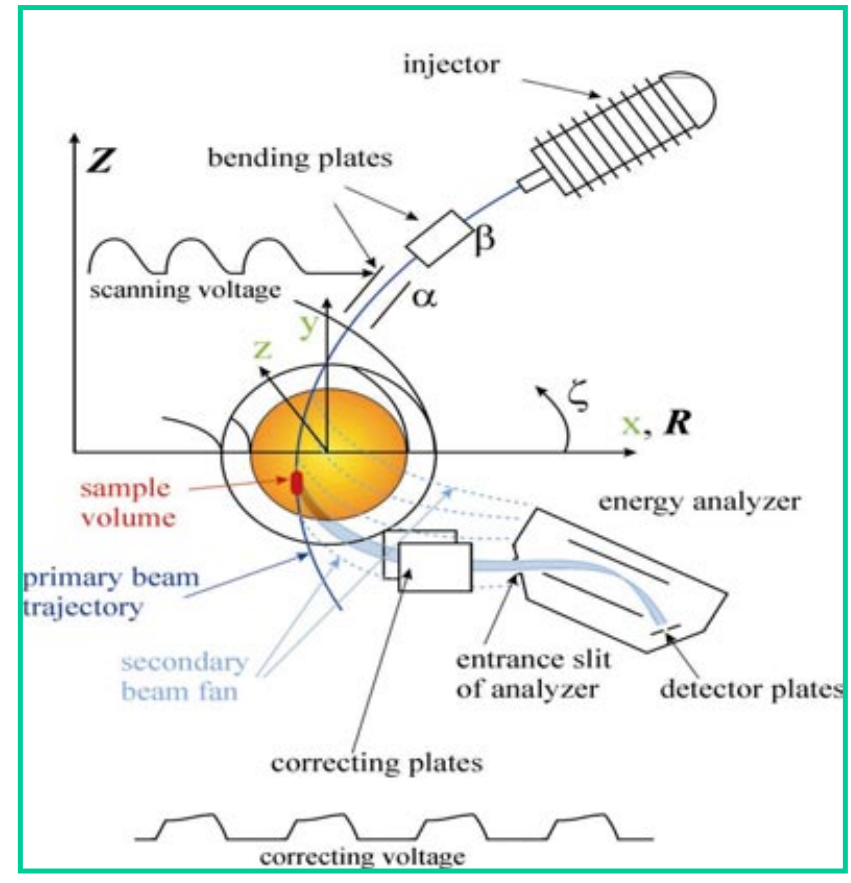

Fig. 1. Basic principles

\section{Main objectives of the WEGA HIBP project.}

The idea of the WEGA HIBP project is to provide the plasma potential measurements in the reasonably wide plasma area, which can give various contributions for the WEGA scientific program. The main objective is the radial profiles of the plasma electric potential and electron densiy investigations. With the measurements of plasma electric potential (mean profiles and fluctuations) HIBP can contribute to the physical understanding of edge (H-mode) and Internal transport barriers (ITB). Density profile can be reconstructed from the secondary beam intensity profile.

\section{WEGA stellarator short}

information $l=2 n=5 \operatorname{Btor}(0) \sim$ up to $0.5 \mathrm{~T} ; R=72 \mathrm{~cm} ; a=19 \mathrm{~cm}$.

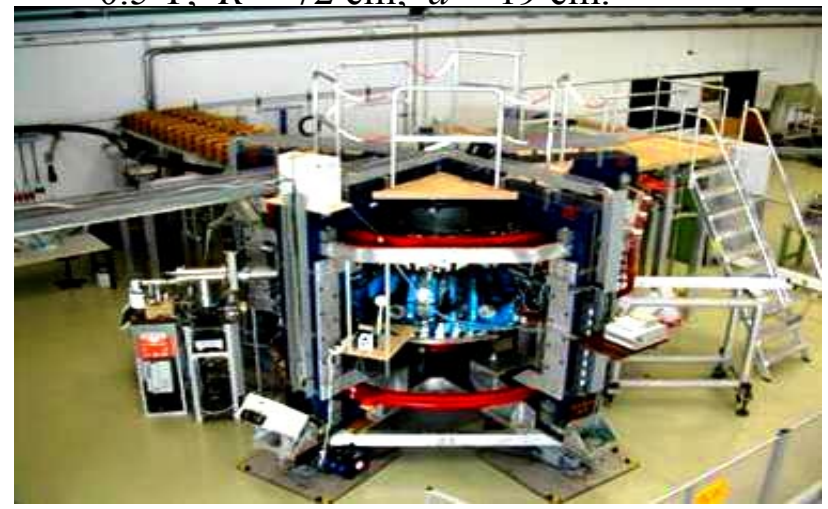

Fig.2. Overview of the WEGA stellarator

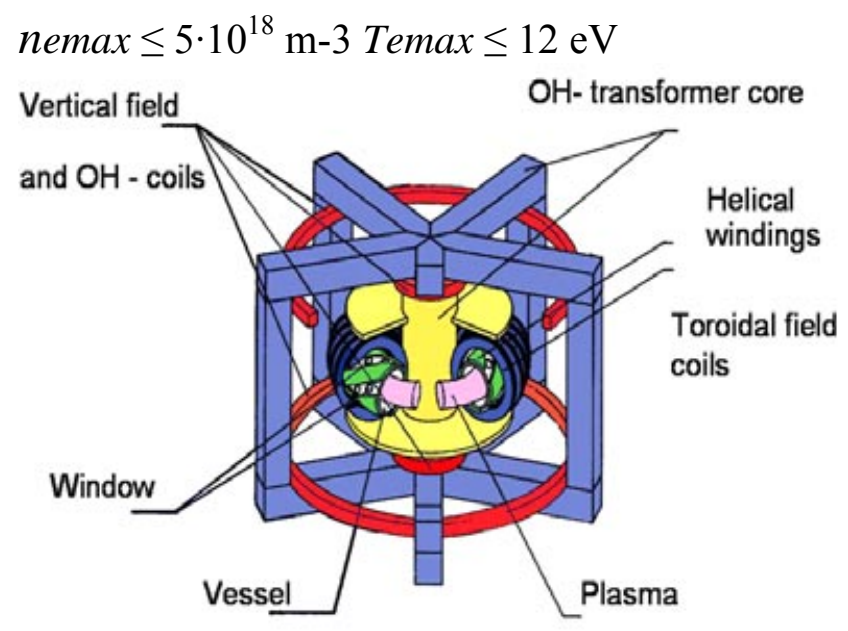

Fig. 3. WEGA stellarator diagram

\section{3D trajectory calculations and optimization of the WEGA features}


The main goals of the HIBP optimization was: (i) finding the probing beam properties (energy, angle of injection ion, mass and so on), providing the detector greed covering maximal plasma cross section. (ii) minimization of the secondary beam trajectories dispersion at the vicinity of the analyzer entrance slit; (iii) choosing the probing pattern in order to optimize (minimize) the beam energy range;(iv) finding an opportunity to carry out the probing beam through existing diagnostic ports of the WEGA vacuum chamber. The complexity of the stellarator magnetic configuration was defined a strong three dimensional nature of the probing beam trajectories The trajectory calculation code solves the 3D equation of motion in the magnetic and electric fields for probing ions. . The resulting magnetic field from all magnetic elements of WEGA stellarator was used for a calculation of primary and secondary ions' trajectories. The system of equations for particles motion in electromagnetic field was solved by the Runge-Kutta method with certain accuracy:[2]

The example of the trajectories in three planes for the secondaries created along the one primary beam trajectory is shown in Fig. 4 for one of the operational regime of the facility. The corresponding detector grid which is projected into analyzer entrance slit by changing the energy and angle of primary $\mathrm{Na}^{+}$beam is shown in Fig 5. The search of the best location of the collection point shows impossibility to obtain only one point for all operational regimes without the degradation of the detector grids or a strong changing of the beam energy range. A large angular range is present on both the primary and secondary beam lines due to the fact that the poloidal and toroidal magnetic fields have comparable strengths.

This results in two activ beam control systems for primary and secondary beam lines[3] with significant requiremqnts in two dimensions. It was developed a procedure for calculating sweep performance including stray magnetic fields. And, based on these calculations, it was designed a two systems for active trajectories control.Fig.4.

\section{HIBP diagnostic equpments for WEGA}

The injection system of WEGA Fig.7 is built on the base of $100 \mathrm{keV}$ sectional accelerating tube with solid-state thermoionic ion source and three electrode extraction-focus system. The diagnostic beam of ions (namely $\mathrm{Na}+$ ) with energy up to $100 \mathrm{keV}$, intensity about $50 \mathrm{mkA}, 5 \mathrm{~mm}$ diameter is received at the entrance into upper $\mathrm{C}$ diagnostic port (1.5 meters from the end of the accelerating tube). The ion optic properties of the injection system was numerically simulated by SIMON* code. The injection system operates in the open air environment. The HV tests did not indicate any sparking during accelerator operation.

The followed primary beam line includes two modules with two pair of the coplanar electrostatic sweep plates for the active control of the primary beam, Faraday cup (introduced manually) and shifted at $10 \mathrm{~mm}$ from the beam axis and four complanar wire detectors. The active beam control by the second pair of sweep plates is extremely important due to three-dimensional nature of beam trajectories in the magnetic fields of TJ-II. This control is realised by programmable drive of the electrostatic plates with TREK* Mod. 609D-6 $(0- \pm 4 \mathrm{kV}, 5 \mathrm{~mA})$ and Mod. $\quad(0- \pm 10$ $\mathrm{kV}, 5 \mathrm{~mA}$ ) HV power amplifiers. 
$\mathrm{Na}+$ ions $\quad \mathrm{B}(0)=0.5 \mathrm{~T}$

Toroidal coil current $=3.4 \mathrm{kA}$

Helix 1 current $=-4.8 \mathrm{kA}$

Helix2 current $=4.8 \mathrm{kA}$

Vertical coil current $=0.11 \mathrm{kA}$

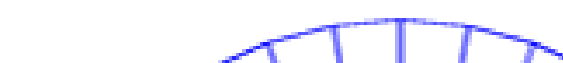

Alfa scan

plates
Injection

point

Injection

point

Detection point
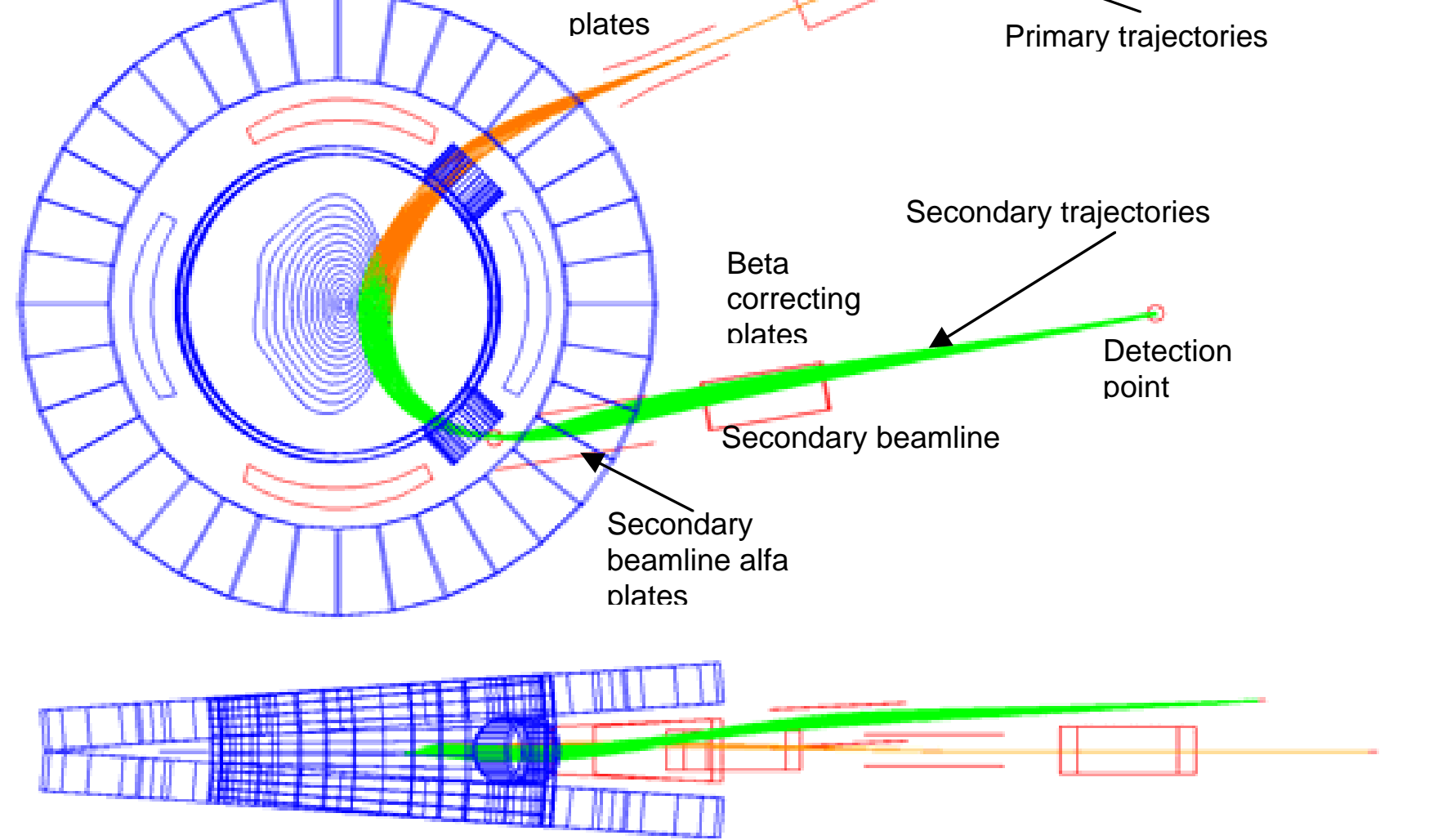

Fig. 4. Trajectory calculation for C+ C- ports :above- XY plane; below-XZ-plane

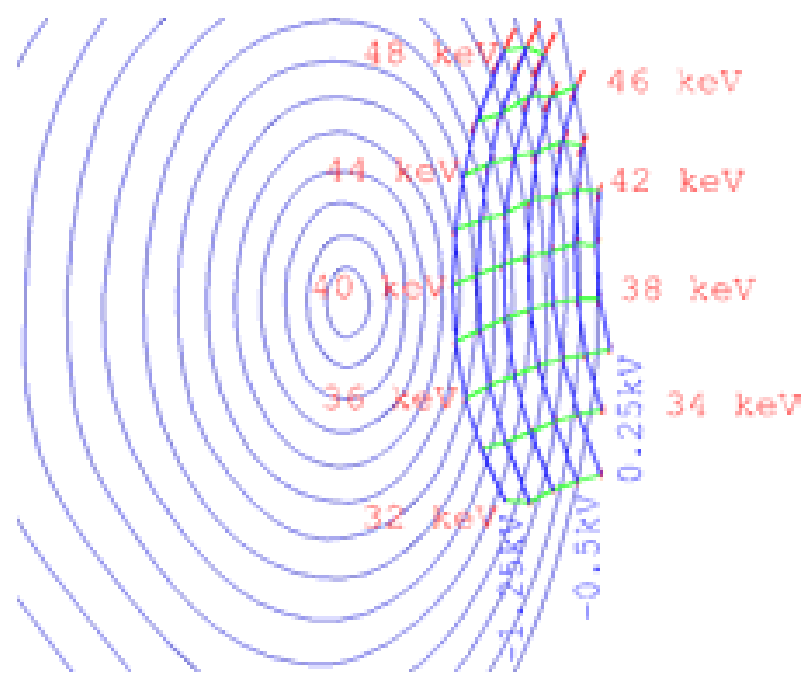

Fig. 5. Detector grid

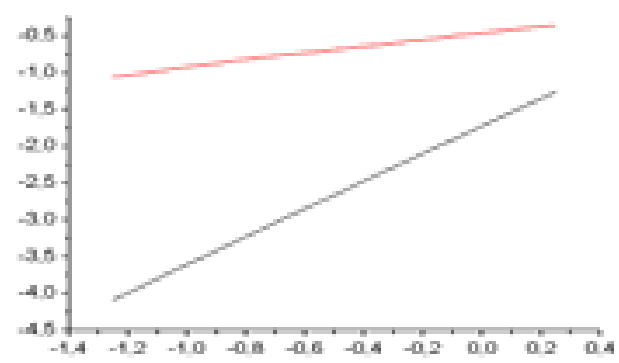

Fig.6. Control voltages

-- equal scan voltage(angle) detector lines

-- equal energy detector lines 
Measurements of plasma potential by HIBP are one of the most difficult problems. These measurements are equivalent to the measurements of the secondary ions energy with resolution $\Delta \mathrm{E} / \mathrm{E}_{\mathrm{b}} \leq 10^{-4}$ where $\mathrm{E}_{\mathrm{b}}$ is probing beam energy. The $30^{0}$ Proca-Green electrostatic energy analyzer [4] was built and calibrated on test facility Fig. 8. Indispensable resolution in the analyzers of this tipe is provided by the differential detection of the secondary beam intensity on split detector. Relation for the value of plasma potential is:

$$
\Phi_{p l}=2 U_{a}\left(\delta i F+G_{a}\right)-U_{b}
$$

where $U_{b}$ and $U_{a}$ are the accelerator and analyser voltages, $\delta i=\left(i_{t}-i_{b}\right) /\left(i_{t}+i_{b}\right)$ is the normalised difference of the currents on the top $\left(i_{t}\right)$ and bottom $\left(i_{b}\right)$ split plates, $G_{a}$ and $F$ are the analyzer gain and dynamic coefficient respectively. They are the function of the analyzer geometry.

The analyzer design for TJ-II is characterized by the absence of guard rings The high uniformity of the electric field inside analyzer $\left(10^{-5}\right.$ at the middleplane) is achieved by a Rogovsky configuration of the top HV electrode arranged symmetrically inside a specially shaped shield kept at earth potential.

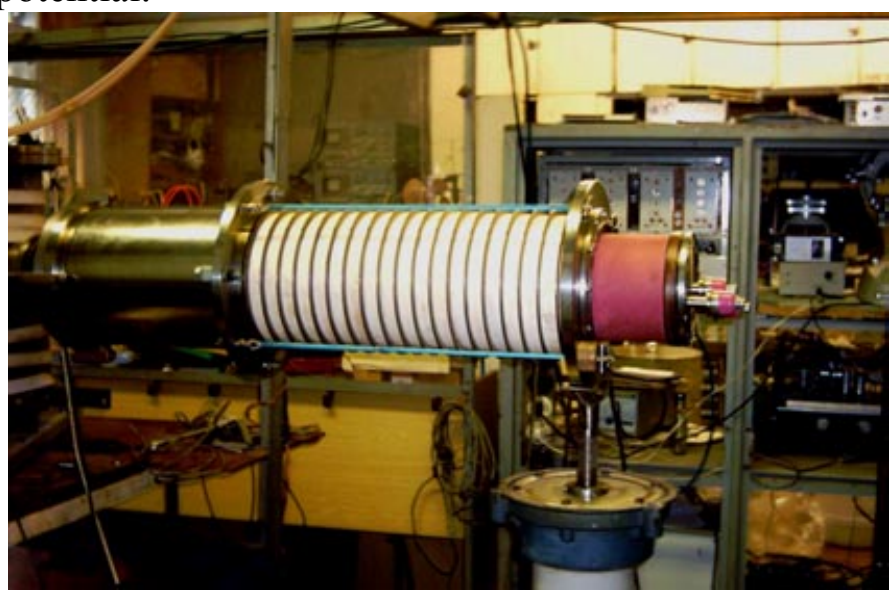

Fig.7. $100 \mathrm{keV}$ ion beam injecror

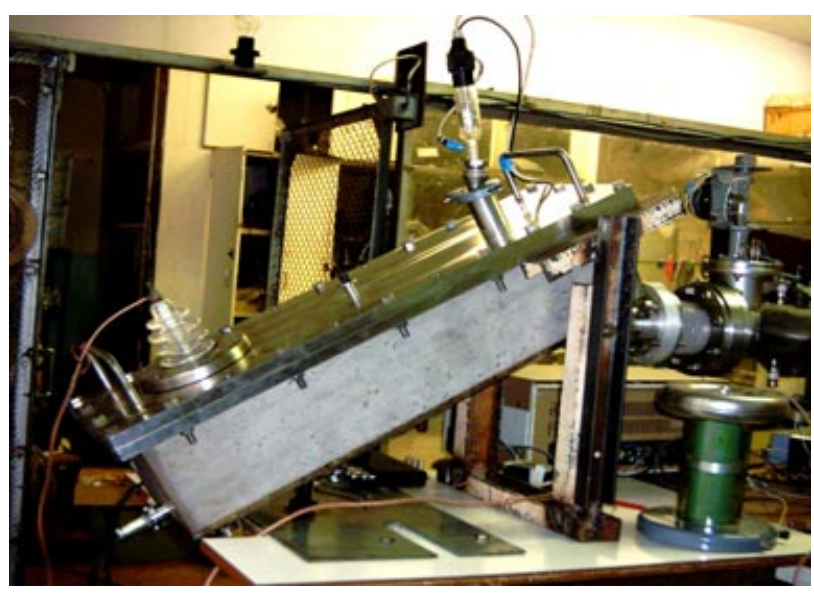

Fig. $8.30^{\circ}$ Proca-Green analyser

\section{Result of the project}

The main calculations of the trajectories were made using singly charged sodium $(\mathrm{Na}+)$ primary ions in the energy range of $30-50 \mathrm{keV}$.

WEGA HIBP project uses entrance port $\mathrm{C}+$ for an injection of primary ion beam and exit port $\mathrm{C}$ - for a detection of the secondary ions beams, coming out of the plasma. These ports are placed on a toroidal angle $=54 \mathrm{o}$ and are perpendicular to each other. Two variants of HIBP diagnostic for different direction of helical coil current were calculated, as it can be changed, but By using the HIBP installation it is possible to get the plasma edge potential profile by fast voltage scan system in the range of $0.3<<1$ with good spatial resolution $(0.3 \mathrm{~cm}$ or less for the $4 \mathrm{~mm}$ detector entrance slit).

Five electrostatic deflection plates of primary and secondary beamlines were used in calculation for scanning and correcting the beam motion. The necessary control voltages on the plates were also calculated.Fig.9. 


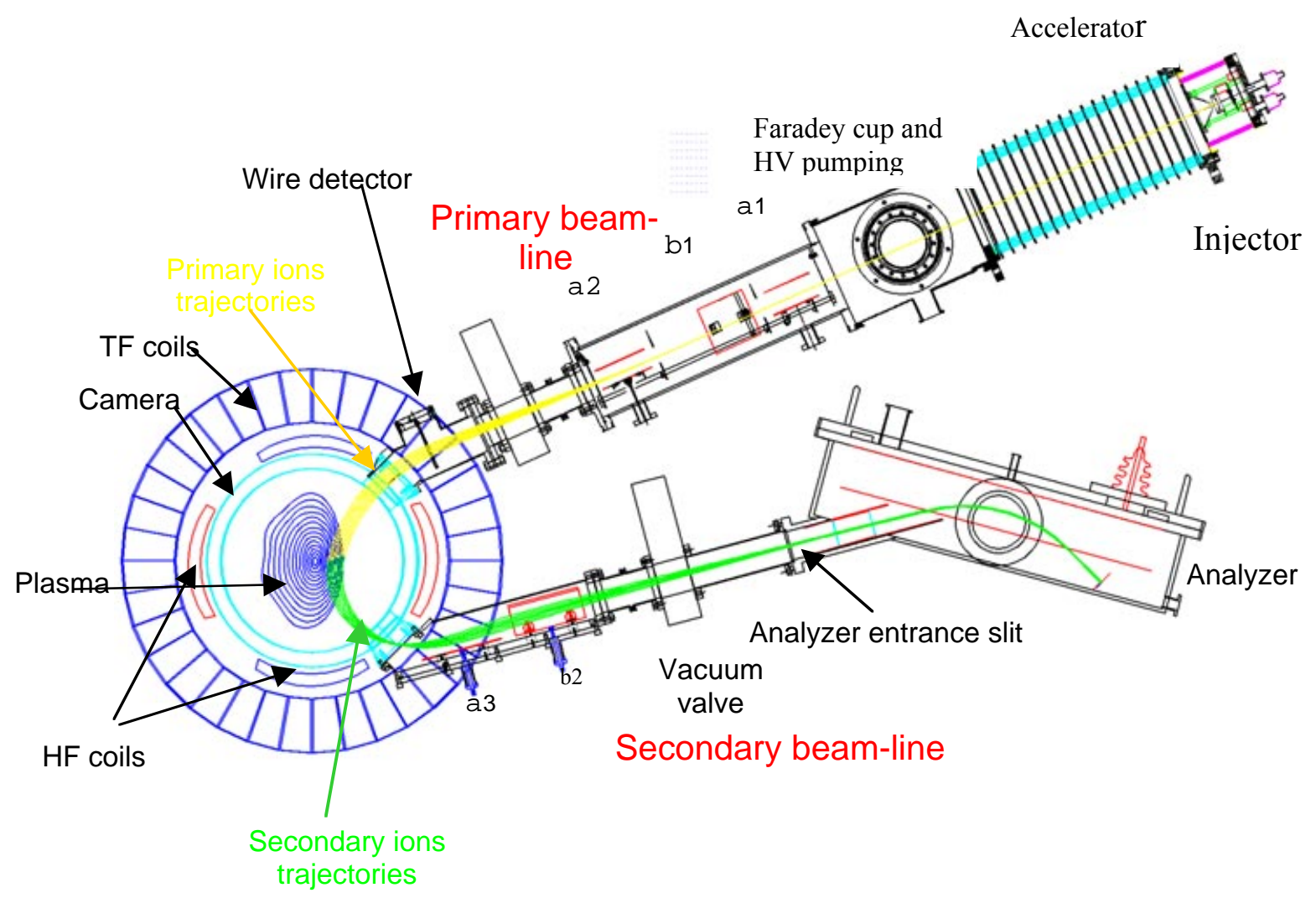

Fig.9.

\section{Conclusions.}

1. The optimized probing scheme for $\mathrm{C}+\mathrm{C}$ - port combination was found. 2. Radial range for sample volume for $\{0.5 \mathrm{~T}, 40 \mathrm{keV}\}$ is $0.3<\rho<1$, the geometrical limitations avoid us to reach the very center. 3 . Toroidal focusing of the secondary trajectories will lead to necessity to construct rather complex secondary beamline.4. Hardware of the HIBP for WEGA was manufactured and tested.

2.

Acknowledgements: The work is supported by Grants INTAS 2001-056 and 20010593, and STCU Grant P-202, 2005-

\section{References}

1. IEEE Trans on Plasma Science, Vol. 22, No.4

2 .V.E.Bykov, L.I.Krupnik, A.V.Melnikov, I.S.Nedzelskij, A.V.Khodyachih, B.A.Shevchuk Calculation of trajectories of heavy ion probe beams in stellarator type instulations "URAGAN-2", "URAGAN-3" and "URAGAN-2M", Preprint KhIPT 88-9, Kharkov, KhIPT AS UkSSR, 1988.

3. J. Lei, T.P. Crowly et. al. Rev. Sci. Instrum. Vol. 70, No.1, p.967, 1999.

4. T.S.Green and G.A.Proca, Rev. Sci. Instrum. Vol. 41, No.10, p.1409, 1970 